\title{
Depression and Microbiome-Study on the Relation and Contiguity between Dogs and Humans
}

\author{
Elisabetta Mondo ${ }^{1, *}$, Alessandra De Cesare ${ }^{1}$, Gerardo Manfreda ${ }^{2}$, Claudia Sala ${ }^{3}(\mathbb{D}$, \\ Giuseppe Cascio ${ }^{1}$, Pier Attilio Accorsi ${ }^{1}$, Giovanna Marliani ${ }^{1}$ (I) and Massimo Cocchi ${ }^{1}$ \\ 1 Department of Veterinary Medical Science, University of Bologna, Via Tolara di Sopra 50, 40064 Ozzano \\ Emilia, Italy; alessandra.decesare@unibo.it (A.D.C.); giuseppe.cascio@unibo.it (G.C.); \\ pierattilio.accorsi@unibo.it (P.A.A.); giovanna.marliani@studio.unibo.it (G.M.); \\ massimo.cocchi@unibo.it (M.C.) \\ 2 Department of Agricultural and Food Sciences, University of Bologna, Via del Florio 2, 40064 Ozzano Emilia, \\ Italy; gerardo.manfreda@unibo.it \\ 3 Department of Physics and Astronomy, Alma Mater Studiorum, University of Bologna, 40126 Bologna, Italy; \\ claudia.sala3@unibo.it \\ * Correspondence: elisabetta.mondo2@unibo.it; Tel.: +39-051-209-7329
}

Received: 22 November 2019; Accepted: 7 January 2020; Published: 13 January 2020

\begin{abstract}
Behavioral studies demonstrate that not only humans, but all other animals including dogs, can suffer from depression. A quantitative molecular evaluation of fatty acids in human and animal platelets has already evidenced similarities between people suffering from depression and German Shepherds, suggesting that domestication has led dogs to be similar to humans. In order to verify whether humans and dogs suffering from similar pathologies also share similar microorganisms at the intestinal level, in this study the gut-microbiota composition of 12 German Shepherds was compared to that of 15 dogs belonging to mixed breeds which do not suffer from depression. Moreover, the relation between the microbiota of the German Shepherd's group and that of patients with depression has been investigated. The results indicate that the German Shepherd's gut-microbiota has a different composition compared to other dog breeds and is characterized by microbial groups identified in humans with depression, highlighting the existence of a "core" microbiota associated with depression.
\end{abstract}

Keywords: gut-microbiota-brain axis; dog depression; human depression; microbiome

\section{Introduction}

In recent years, several studies have shown that depression is not just a human disease but it has a molecular affinity within the animal kingdom [1,2]. Until now, research on depression have mainly been focused on the genetic, behavioral and neurological aspects of the mental illness. However, recent evidences from animal studies have shown that functions of the central nervous system (CNS) are influenced by the gut microbiota and this is also involved in the pathogenesis of mental diseases. This influence happens through inflammation, the hypothalamic-pituitary-adrenal (HPA) axis and by the production of neurotransmission precursors [3]. Such a network has an impact on physiological features affecting mammalian behavior. In the study conducted by Sudo and colleagues, it is showed germ-free (GF) mice had an abnormal functioning of the HPA axis, with higher adrenocorticotropic hormone $(\mathrm{ACTH})$ and corticosterone levels rather than specific pathogen-free (SPF) mice in response to restraint stress. These abnormal hormonal levels normalized after colonization with commensal bacteria [4]. Besides, GF had a lower level of brain-derived neurotrophic factor (BDNF) in the cortex and hippocampus [4]. Furthermore, several studies have found that administration of probiotics improves the health of the host by modulating anxiety phenotypes and stress hormones' response. 
It has been observed that the administration of Lactobacillus rhamnosus (JB-1) and Bacteroides fragilis (NCTC 9343) reduces anxiety-like behavior [5,6]. Moreover, gut bacteria can produce neuroactive substances, such as precursors of monoamine neurotransmitters, which support the communication with the brain, affecting behavior, including anxiety, stress and depression [4,7]. One of the key roles in bidirectional gut-microbiota-brain communication is carried out by tryptophan, which impacts host serotonin and kynurenine levels, influencing both behaviors linked to serotonergic neurotransmission and immune system [8]. All these observations indicate that the gut microbiome and some behavioral patterns may be linked in mammals. In 2006, Cocchi [9] carried out a study on the similarity between the fatty acid profiles of platelets and neurons. Based on this similarity, they were able to identify different mood disorders (Major Depression and Bipolar Disorder) by creating an artificial neural network, whose crucial factors were three fatty acids (i.e., Palmitic Acid, Linoleic Acid, Arachidonic Acid) $[10,11]$. The same study was conducted taking into account different animal species and its most important evidence was the molecular similarity between two particular dog breeds (German Shepherd and Alaskan Malamute) and humans affected by Major Depression and Bipolar Disorder [2,12,13]. Considering the molecular similarity in fatty acids platelets between German Shepherds and humans suffering from depression and the studies on the gut-microbiota of the latter, the aim of this research was to analyze the gut-microbiota composition of German Shepherd in comparison to other canine mixed breeds not described as the suffering of depression disease.

\section{Materials and Methods}

\subsection{Animals}

Twenty-seven dogs were recruited from dog breeders (12 German Shepherds GS) and private owners (15 mixed breeds MB), whit an age between $2-8$ years. At the sampling time of their faces, all dogs were clinically healthy and had not received any medications that could have affected the gut microbiota in the 4 months before the sampling. Besides, all investigated dogs were fed with an industrial diet. The chemical composition of industrial feed was crude protein (18-21\% of total content), crude fat $(8-10 \%)$, crude fiber $(3-5 \%)$, crude ash $(7 \%)$ and $10 \%$ of moisture.

\subsection{DNA Extraction from Stool Samples}

A small sample of feces (i.e., $0.5-2 \mathrm{~g}$ ) was collected from each of the 25 dogs into $2 \mathrm{~mL}$ sterile plastic tubes. The DNA was extracted from each sample using a bead-beating procedure as previously described De Cesare et al. 2017 [14].

\subsection{Library Preparation and Sequencing}

The libraries were prepared and then sequenced as described by De Cesare et al. 2019 [15]. All sequences were analyzed using MG-RAST (http://metagenomics.anl.gov/). After applying the quality control procedure, following the instructions of the MG-RAST manual, the taxonomic classification of the sequencing data was performed by applying the Best Hit Classification method and using the M5RNA database. All metagenomes deposited in MG-RAST are public under the project labelled as Prova Cani (https:/www.mg-rast.org/mgmain.html?mgpage=project\&project=mgp86824) and detailed in Table S1 (Supplement Material).

\subsection{Statistical Analysis}

The reads obtained from each sample were analyzed by using MG-RAST (https:/www.mg-rast. $\mathrm{org} /$ ) and the values of the relative frequency of abundance of each taxonomic level within each animal were compared using the $t$-test of Tukey-Kramer in the software Statistical Analysis of Metagenomic Profile (STAMP) v 2.0.9. $p$ values $<0.05$ were considered statistically significant. Alpha and beta diversity analysis were performed in Python 3.6.3. Alpha diversity was calculated using an in-house pipeline that computes the indices from the normalized read counts. Alpha diversities of different 
animals were compared using the Student's $t$-test. $p$ values were adjusted for multiple testing using the Benjamini-Hochberg procedure. Bray-Curtis beta diversity and Principal Coordinate analysis were computed using scikit-bio 0.4.2. Heat maps and complete linkage clustering dendrograms based on beta diversity were obtained with scipy 1.1.0.

\section{Results}

The most abundant phyla identified in the dog feces were represented by Firmicutes, Bacteroidetes and Fusobacteria (Table 1). The phylum Bacteroidetes was significantly higher in MB in comparison to GS, while Proteobacteria was significantly higher in GS rather than in MB (Table 1). The most abundant classes were Bacteroidia, Clostridia and Fusobacteria (Table 2). Among the classes with a relative mean frequency of abundance $>0.1 \%$, Bacilli, Deltaproteobacteria and Gammaproteobacteria were significantly higher in GS in comparison to MB. On the contrary, Bacteroidia and Negativicutes were significantly higher in MB rather than in GS (Table 2). The most abundant orders were Bacteroidales, Clostridiales, Lactobacillales and Fusobacteriales. The order Bacteroidales, as well as Selenomonadales, was significantly higher in MB than in GS, while Lactobacillales and Desulfovibrionales were significantly higher in GS in comparison to MB (Table 3).

Table 1. Mean relative frequency of abundance (\%) and corresponding standard deviation (SD) (\%) of the phyla identified in the caeca of mixed breeds (MB) and German Shepherd (GS) dogs. The species indicated are those showing a mean relative frequency of abundance $\geq 0.1 \%$ in at least one group. $p$ values in bold are those significantly different.

\begin{tabular}{cccccc}
\hline \multicolumn{2}{c}{ MB } & \multicolumn{3}{c}{ GS } \\
\hline Phylum & Mean (\%) & std. dev. (\%) & Mean (\%) & PT std. dev. (\%) & $p$ Values \\
\hline Firmicutes & 55.247 & 13.880 & 55.695 & 14.042 & 0.937 \\
Fusobacteria & 16.599 & 9.218 & 11.296 & 6.569 & 0.106 \\
Bacteroidetes & 21.306 & 11.162 & 10.742 & 10.611 & 0.024 \\
Actinobacteria & 3.608 & 2.868 & 3.453 & 1.827 & 0.871 \\
Proteobacteria & 0.540 & 0.293 & 1.916 & 0.756 & 0.000 \\
\hline
\end{tabular}

Table 2. Mean relative frequency of abundance (\%) and corresponding standard deviation (SD) (\%) of the classes identified in the caeca of mixed breeds (MB) and German Shepherd (GS) dogs. The species indicated are those showing a mean relative frequency of abundance $\geq 0.1 \%$ in at least one group. $p$ values in bold are those who are significantly different.

\begin{tabular}{cccccc}
\hline \multicolumn{7}{c}{ MB } & GS \\
\hline Class & Mean (\%) & std. dev. (\%) & Mean (\%) & std. dev. (\%) & $p$ Values \\
\hline Actinobacteria & 3.608 & 2.868 & 3.453 & 1.827 & 0.871 \\
Bacteroidia & 21.298 & 11.157 & 10.724 & 10.603 & 0.023 \\
Clostridia & 39.318 & 11.190 & 33.425 & 8.456 & 0.146 \\
Bacilli & 2.019 & 1.476 & 14.761 & 7.947 & 0.000 \\
Erysipelotrichi & 8.471 & 7.689 & 4.664 & 3.423 & 0.113 \\
Negativicutes & 5.439 & 3.881 & 2.846 & 1.723 & 0.037 \\
Fusobacteria & 16.599 & 9.218 & 11.296 & 6.569 & 0.106 \\
Deltaproteobacteria & 0.158 & 0.217 & 0.973 & 0.504 & 0.000 \\
Gammaproteobacteria & 0.161 & 0.169 & 0.825 & 0.681 & 0.008 \\
Epsilonproteobacteria & 0.161 & 0.225 & 0.022 & 0.016 & 0.037 \\
\hline
\end{tabular}

The most abundant families in the tested feces were Prevotellaceae, Bacteroidaceae, Clostridiaceae and Fusobacteriaceae (Table 4). The abundance of the families Bacteroidaceae, Ruminococcaceae and Veillonellaceae were significantly higher in MB than in GS. On the contrary Microbacteriaceae, Streptococcaceae, Lactobacillaceae, Aerococcaceae and Enterococcaceae were significantly higher in GS in comparison to MB (Table 4). 
Table 3. Mean relative frequency of abundance (\%) and corresponding standard deviation (SD) (\%) of the orders identified in the caeca of mixed breeds (MB) and German Shepherd (GS) dogs. The species indicated are those showing a mean relative frequency of abundance $\geq 0.1 \%$ in at least one group. $p$ values in bold are those significantly different.

\begin{tabular}{cccccc}
\hline \multicolumn{2}{c}{ MB } & \multicolumn{3}{c}{ GS } \\
\hline Order & Mean (\%) & std. dev. (\%) & Mean (\%) & std. dev. (\%) & $p$ Values \\
\hline Coriobacteriales & 3.502 & 2.839 & 2.471 & 0.991 & 0.222 \\
Actinomycetales & 0.081 & 0.044 & 0.946 & 1.758 & 0.131 \\
Bacteroidales & 21.298 & 11.157 & 10.724 & 10.603 & 0.023 \\
Clostridiales & 39.302 & 11.187 & 33.404 & 8.453 & 0.146 \\
Lactobacillales & 1.182 & 1.403 & 13.530 & 7.905 & 0.000 \\
Erysipelotrichales & 8.471 & 7.689 & 4.664 & 3.423 & 0.113 \\
Selenomonadales & 5.439 & 3.881 & 2.846 & 1.723 & 0.037 \\
Bacillales & 0.837 & 0.635 & 1.231 & 0.682 & 0.153 \\
Fusobacteriales & 16.599 & 9.218 & 11.296 & 6.569 & 0.106 \\
Desulfovibrionales & 0.111 & 0.147 & 0.952 & 0.503 & 0.000 \\
Enterobacteriales & 0.028 & 0.035 & 0.395 & 0.289 & 0.001 \\
Aeromonadales & 0.121 & 0.164 & 0.226 & 0.273 & 0.273 \\
\hline
\end{tabular}

Table 4. Mean relative frequency of abundance (\%) and corresponding standard deviation (SD) (\%) of the families identified in the caeca of mixed breeds (MB) and German Shepherd (GS) dogs. The species indicated are those showing a mean relative frequency of abundance $\geq 0.1 \%$ in at least one group. $p$ values in bold are those who are significantly different.

\begin{tabular}{|c|c|c|c|c|c|}
\hline \multirow[b]{2}{*}{ Family } & \multicolumn{2}{|c|}{ MB } & \multicolumn{2}{|c|}{ GS } & \multirow[b]{2}{*}{$p$ Values } \\
\hline & Mean (\%) & std. dev. (\%) & Mean (\%) & std. dev. $(\%)$ & \\
\hline Coriobacteriaceae & 3.502 & 2.839 & 2.471 & 0.991 & 0.222 \\
\hline Microbacteriaceae & 0.030 & 0.029 & 0.587 & 0.907 & 0.067 \\
\hline Micrococcaceae & 0.002 & 0.002 & 0.117 & 0.377 & 0.333 \\
\hline Corynebacteriaceae & 0.002 & 0.001 & 0.042 & 0.104 & 0.222 \\
\hline Prevotellaceae & 11.113 & 9.461 & 6.368 & 6.892 & 0.160 \\
\hline Bacteroidaceae & 10.010 & 7.919 & 3.891 & 5.036 & 0.027 \\
\hline Porphyromonadaceae & 0.165 & 0.209 & 0.448 & 0.315 & 0.019 \\
\hline Clostridiaceae & 15.448 & 7.344 & 14.324 & 4.907 & 0.651 \\
\hline Ruminococcaceae & 8.874 & 2.640 & 6.695 & 2.128 & 0.031 \\
\hline Erysipelotrichaceae & 8.471 & 7.689 & 4.664 & 3.423 & 0.113 \\
\hline Veillonellaceae & 3.811 & 3.828 & 0.908 & 0.560 & 0.014 \\
\hline Lachnospiraceae & 3.810 & 2.160 & 2.799 & 1.241 & 0.155 \\
\hline Eubacteriaceae & 1.990 & 1.349 & 1.766 & 1.604 & 0.714 \\
\hline Acidaminococcaceae & 1.628 & 2.117 & 1.938 & 1.537 & 0.675 \\
\hline Streptococcaceae & 0.608 & 1.407 & 3.440 & 2.936 & 0.010 \\
\hline Paenibacillaceae & 0.525 & 0.472 & 0.640 & 0.371 & 0.501 \\
\hline Lactobacillaceae & 0.417 & 0.442 & 8.616 & 7.893 & 0.005 \\
\hline Bacillaceae & 0.281 & 0.383 & 0.222 & 0.085 & 0.584 \\
\hline Peptostreptococcaceae & 0.215 & 0.127 & 0.148 & 0.063 & 0.097 \\
\hline Aerococcaceae & 0.132 & 0.182 & 0.640 & 0.344 & 0.000 \\
\hline Peptococcaceae & 0.091 & 0.069 & 0.334 & 0.201 & 0.002 \\
\hline Enterococcaceae & 0.015 & 0.035 & 0.661 & 0.368 & 0.000 \\
\hline Thermoactinomycetaceae & 0.010 & 0.010 & 0.136 & 0.248 & 0.120 \\
\hline Leuconostocaceae & 0.008 & 0.017 & 0.131 & 0.152 & 0.021 \\
\hline $\begin{array}{c}\text { Clostridiales Family XII. Incertae } \\
\text { Sedis }\end{array}$ & 0.008 & 0.020 & 0.177 & 0.116 & 0.001 \\
\hline Listeriaceae & 0.001 & 0.001 & 0.180 & 0.577 & 0.324 \\
\hline Fusobacteriaceae & 16.599 & 9.218 & 11.296 & 6.569 & 0.106 \\
\hline Helicobacteraceae & 0.124 & 0.217 & 0.003 & 0.002 & 0.056 \\
\hline Desulfohalobiaceae & 0.110 & 0.147 & 0.950 & 0.503 & 0.000 \\
\hline Succinivibrionaceae & 0.090 & 0.155 & 0.223 & 0.272 & 0.168 \\
\hline Enterobacteriaceae & 0.028 & 0.035 & 0.395 & 0.289 & 0.001 \\
\hline
\end{tabular}


The most abundant genera identified in the tested samples were Prevotella, Bacteroides, Clostridium and Fusobacterium. Bacteroides, Megamonas and Selenomonas were significantly higher in MB in comparison to GS, while Desulfonauticus, Streptococcus, Lactobacillus, Aerococcus and Enterococcus, were significantly higher in GS compared to MB (Table 5). The most abundant species were Clostridium bifermentans, Fusobacterium mortiferum, Lactobacillus murinus, Prevotella copri and Blautia sp. Ser8. In particular, the species Lactobacillus murinus and Blautia sp. Ser8 were significantly higher in GS in comparison to MB, while Clostridium bifermentans, Fusobacterium mortiferum and Prevotella copri were significantly higher in MB rather than in GS (Table 6). The species with a $p$ value $\geq 0.01 \%$ were uncultured bacteria, Bacteroides plebeius, Fusobacterium equinum and Clostridium scindens. Among all, the uncultured bacteria were significantly higher in GS in comparison to MB, while Bacteroides uniformis was significantly lower in GS rather than in MB (Table 6).

Table 5. Mean relative frequency of abundance (\%) and corresponding standard deviation (SD) (\%) of the genera identified in the caeca of mixed breeds (MB) and German Shepherd (GS) dogs. The species indicated are those showing a mean relative frequency of abundance $\geq 0.1 \%$ in at least one group. $p$ values in bold are those who are significantly different.

\begin{tabular}{|c|c|c|c|c|c|}
\hline & \multicolumn{2}{|c|}{ MB } & \multicolumn{2}{|c|}{ GS } & \multirow[b]{2}{*}{$p$ Values } \\
\hline Genus & Mean (\%) & std. dev. (\%) & Mean (\%) & std. dev. (\%) & \\
\hline Microbacterium & 0.008 & 0.019 & 0.497 & 0.788 & 0.064 \\
\hline Anaerobiospirillum & 0.090 & 0.155 & 0.223 & 0.272 & 0.168 \\
\hline Paenibacillus & 0.521 & 0.471 & 0.634 & 0.374 & 0.512 \\
\hline Bacillus & 0.200 & 0.390 & 0.160 & 0.088 & 0.718 \\
\hline Thermoactinomyces & 0.009 & 0.010 & 0.133 & 0.246 & 0.124 \\
\hline Prevotella & 11.078 & 9.463 & 6.317 & 6.814 & 0.157 \\
\hline Bacteroides & 10.010 & 7.919 & 3.891 & 5.036 & 0.027 \\
\hline Parabacteroides & 0.080 & 0.199 & 0.119 & 0.138 & 0.570 \\
\hline Porphyromonas & 0.046 & 0.049 & 0.157 & 0.118 & 0.011 \\
\hline Barnesiella & 0.023 & 0.033 & 0.118 & 0.111 & 0.017 \\
\hline Helicobacter & 0.124 & 0.217 & 0.003 & 0.002 & 0.056 \\
\hline Clostridium & 15.007 & 7.362 & 13.934 & 4.942 & 0.668 \\
\hline Blautia & 6.746 & 3.580 & 5.307 & 2.441 & 0.245 \\
\hline Ruminococcus & 5.411 & 2.917 & 3.892 & 0.998 & 0.086 \\
\hline Faecalibacterium & 3.290 & 3.013 & 2.432 & 2.158 & 0.415 \\
\hline Eubacterium & 1.987 & 1.350 & 1.753 & 1.586 & 0.699 \\
\hline Hespellia & 1.141 & 0.728 & 0.778 & 0.265 & 0.101 \\
\hline Robinsoniella & 0.519 & 0.513 & 0.442 & 0.501 & 0.707 \\
\hline Coprococcus & 0.514 & 0.846 & 0.222 & 0.625 & 0.331 \\
\hline Roseburia & 0.479 & 0.527 & 0.133 & 0.127 & 0.031 \\
\hline Butyrivibrio & 0.369 & 0.485 & 0.369 & 0.132 & 0.999 \\
\hline Lachnospira & 0.246 & 0.517 & 0.098 & 0.119 & 0.316 \\
\hline Peptostreptococcus & 0.215 & 0.127 & 0.148 & 0.063 & 0.097 \\
\hline Alkaliphilus & 0.201 & 0.427 & 0.093 & 0.097 & 0.372 \\
\hline Syntrophococcus & 0.105 & 0.326 & 0.001 & 0.001 & 0.253 \\
\hline Ethanoligenens & 0.099 & 0.139 & 0.231 & 0.220 & 0.101 \\
\hline Butyricicoccus & 0.096 & 0.063 & 0.122 & 0.064 & 0.337 \\
\hline Sarcina & 0.082 & 0.305 & 0.162 & 0.364 & 0.569 \\
\hline Peptococcus & 0.037 & 0.064 & 0.222 & 0.102 & 0.000 \\
\hline Fusibacter & 0.008 & 0.020 & 0.177 & 0.116 & 0.001 \\
\hline Collinsella & 2.176 & 1.793 & 1.329 & 0.544 & 0.113 \\
\hline Slackia & 0.926 & 0.759 & 0.726 & 0.365 & 0.394 \\
\hline Enterorhabdus & 0.233 & 0.197 & 0.222 & 0.068 & 0.845 \\
\hline Atopobium & 0.140 & 0.115 & 0.130 & 0.065 & 0.797 \\
\hline Desulfonauticus & 0.102 & 0.147 & 0.923 & 0.495 & 0.000 \\
\hline Escherichia & 0.009 & 0.023 & 0.249 & 0.156 & 0.000 \\
\hline
\end{tabular}


Table 5. Cont.

\begin{tabular}{cccccc}
\hline & \multicolumn{3}{c}{ MB } & \multicolumn{3}{c}{ GS } \\
\hline Genus & Mean (\%) & std. dev. (\%) & Mean (\%) & std. dev. (\%) & $p$ Values \\
\hline Catenibacterium & 1.503 & 2.894 & 0.680 & 0.972 & 0.333 \\
Erysipelothrix & 0.481 & 0.827 & 0.436 & 0.334 & 0.853 \\
Holdemania & 0.100 & 0.293 & 0.139 & 0.128 & 0.667 \\
Fusobacterium & 16.573 & 9.213 & 11.284 & 6.562 & 0.107 \\
Streptococcus & 0.600 & 1.406 & 3.403 & 2.930 & 0.011 \\
Lactobacillus & 0.417 & 0.442 & 8.611 & 7.888 & 0.005 \\
Aerococcus & 0.129 & 0.181 & 0.627 & 0.344 & 0.001 \\
Enterococcus & 0.014 & 0.035 & 0.651 & 0.362 & 0.000 \\
Megamonas & 2.671 & 3.011 & 0.432 & 0.503 & 0.015 \\
Phascolarctobacterium & 1.176 & 1.325 & 1.365 & 1.042 & 0.692 \\
Selenomonas & 1.125 & 0.903 & 0.463 & 0.387 & 0.023 \\
Acidaminococcus & 0.452 & 0.848 & 0.572 & 0.529 & 0.668 \\
\hline
\end{tabular}

Table 6. Mean relative frequency of abundance (\%) and corresponding standard deviation (SD) (\%) of the species identified in the caeca of mixed breeds (MB) and German Shepherd (GS) dogs. The species indicated are those showing a mean relative frequency of abundance $\geq 0.5 \%$ in at least one group. $p$ values in bold are those who are significantly different.

\begin{tabular}{|c|c|c|c|c|c|}
\hline & & IB & & GS & \\
\hline Species & Mean (\%) & std. dev. (\%) & Mean (\%) & std. dev. (\%) & $p$ Values \\
\hline Phascolarctobacterium sp. YIT 12067 & 1.176 & 1.325 & 1.365 & 1.042 & 0.692 \\
\hline Acidaminococcus fermentans & 0.448 & 0.833 & 0.572 & 0.529 & 0.654 \\
\hline Aerococcus viridans & 0.118 & 0.174 & 0.612 & 0.333 & 0.000 \\
\hline Bacteroides plebeius & 2.619 & 2.635 & 0.370 & 0.456 & 0.007 \\
\hline Bacteroides fragilis & 1.689 & 2.246 & 0.628 & 0.938 & 0.126 \\
\hline Bacteroides stercoris & 0.984 & 0.883 & 0.741 & 1.515 & 0.643 \\
\hline Bacteroides coprocola & 0.963 & 0.790 & 0.390 & 0.460 & 0.033 \\
\hline Bacteroides vulgatus & 0.907 & 2.099 & 0.055 & 0.121 & 0.152 \\
\hline Bacteroides uniformis & 0.785 & 0.844 & 0.184 & 0.204 & 0.021 \\
\hline Bacteroides ovatus & 0.506 & 0.464 & 0.360 & 0.565 & 0.497 \\
\hline Clostridium bifermentans & 5.080 & 3.341 & 3.625 & 1.436 & 0.158 \\
\hline Clostridium sordellii & 3.167 & 3.022 & 3.073 & 1.121 & 0.916 \\
\hline Clostridium bartlettii & 1.769 & 1.213 & 0.904 & 0.366 & 0.022 \\
\hline Clostridium scindens & 1.142 & 1.020 & 0.230 & 0.120 & 0.005 \\
\hline Clostridium hiranonis & 0.979 & 1.494 & 0.329 & 0.980 & 0.203 \\
\hline Clostridium perfringens & 0.226 & 0.370 & 1.738 & 1.664 & 0.012 \\
\hline Clostridium aminobutyricum & 0.044 & 0.120 & 0.813 & 0.551 & 0.001 \\
\hline Collinsella intestinalis & 1.840 & 1.534 & 1.121 & 0.482 & 0.116 \\
\hline Slackia heliotrinireducens & 0.890 & 0.734 & 0.684 & 0.363 & 0.370 \\
\hline Desulfonauticus autotrophicus & 0.101 & 0.147 & 0.923 & 0.495 & 0.000 \\
\hline Clostridium ramosum & 1.684 & 2.043 & 0.499 & 0.608 & 0.055 \\
\hline Catenibacterium mitsuokai & 1.503 & 2.894 & 0.680 & 0.972 & 0.333 \\
\hline Eubacterium biforme & 1.060 & 1.492 & 0.879 & 0.730 & 0.695 \\
\hline Lactobacillus vitulinus & 0.823 & 1.583 & 0.348 & 0.688 & 0.326 \\
\hline Clostridium spiroforme & 0.790 & 0.914 & 0.390 & 0.239 & 0.135 \\
\hline Eubacterium cylindroides & 0.650 & 0.643 & 0.563 & 0.351 & 0.670 \\
\hline Streptococcus pleomorphus & 0.620 & 0.956 & 0.481 & 0.417 & 0.630 \\
\hline Eubacterium fissicatena & 1.079 & 1.098 & 0.397 & 0.176 & 0.037 \\
\hline Fusobacterium nucleatum & 6.030 & 3.870 & 4.852 & 2.987 & 0.398 \\
\hline Fusobacterium mortiferum & 2.156 & 1.684 & 1.167 & 0.892 & 0.072 \\
\hline Fusobacterium varium & 2.109 & 1.455 & 1.945 & 1.408 & 0.779 \\
\hline Fusobacterium ulcerans & 2.002 & 1.457 & 1.547 & 0.961 & 0.359 \\
\hline Fusobacterium equinum & 1.698 & 1.015 & 0.702 & 0.539 & 0.005 \\
\hline Fusobacterium perfoetens & 1.628 & 1.375 & 0.602 & 0.592 & 0.021 \\
\hline Fusobacterium periodonticum & 0.794 & 0.851 & 0.418 & 0.612 & 0.211 \\
\hline Hespellia porcina & 0.682 & 0.472 & 0.451 & 0.191 & 0.112 \\
\hline Robinsoniella peoriensis & 0.519 & 0.513 & 0.442 & 0.501 & 0.707 \\
\hline
\end{tabular}


Table 6. Cont.

\begin{tabular}{cccccc}
\hline & \multicolumn{3}{c}{ MB } & GS & \\
\hline Species & Mean (\%) & std. dev. (\%) & Mean (\%) & std. dev. (\%) & $p$ Values \\
\hline Coprococcus comes & 0.509 & 0.845 & 0.219 & 0.624 & 0.332 \\
Lactobacillus murinus & 0.014 & 0.015 & 5.202 & 5.009 & 0.006 \\
Lactobacillus reuteri & 0.004 & 0.003 & 1.193 & 1.600 & 0.031 \\
Prevotella copri & 5.976 & 5.824 & 2.289 & 2.473 & 0.045 \\
Prevotella intermedia & 1.434 & 1.868 & 1.018 & 1.008 & 0.483 \\
Prevotella oris & 1.024 & 1.700 & 0.325 & 0.556 & 0.166 \\
Prevotella ruminicola & 0.863 & 1.073 & 0.479 & 0.449 & 0.240 \\
Prevotella falsenii & 0.658 & 0.888 & 0.457 & 0.473 & 0.474 \\
Prevotella nigrescens & 0.599 & 0.811 & 1.281 & 2.520 & 0.404 \\
Faecalibacterium prausnitzii & 3.290 & 3.013 & 2.432 & 2.158 & 0.415 \\
Ruminococcus gnavus & 2.838 & 3.070 & 0.955 & 0.322 & 0.038 \\
Ruminococcus sp. 5_1_39BFAA & 0.885 & 1.151 & 0.835 & 0.282 & 0.878 \\
Ruminococcus obeum & 0.815 & 0.997 & 0.649 & 0.309 & 0.565 \\
Ruminococcus torques & 0.542 & 0.663 & 0.566 & 0.163 & 0.897 \\
Ruminococcus gauvreauii & 0.254 & 0.148 & 0.687 & 0.252 & 0.000 \\
Streptococcus agalactiae & 0.247 & 0.633 & 1.630 & 2.194 & 0.065 \\
Blautia sp. Ser8 & 6.484 & 3.417 & 5.052 & 2.480 & 0.236 \\
butyrate-producing bacterium SM4/1 & 1.262 & 1.041 & 1.201 & 0.538 & 0.852 \\
Megamonas hypermegale & 2.671 & 3.011 & 0.432 & 0.503 & 0.015 \\
Selenomonas ruminantium & 0.957 & 0.804 & 0.449 & 0.391 & 0.050 \\
\hline
\end{tabular}

$p$ values of alpha diversity used in the study by different indexes in the tested groups at different taxonomic levels are reported in Table 7 and Figure 1. The Index of median values in the two groups is described in Table 8. According to Bray-Curtis beta diversity and Principal Coordinates Analysis (PCoA), the inter-sample variation highlighted a significant separation between the structural composition of the gut microbiota among the study groups (Figures 2 and 3).

Table 7. $p$ values of alpha diversity calculated by different indexes in the tested groups at different taxonomic levels. $p$ values in bold are those significantly different.

\begin{tabular}{ccccccc}
\hline & Phylum & Class & Order & Family & Genus & Species \\
\hline Simpson & 0.139 & 0.326 & 0.231 & 0.003 & 0.004 & 0.142 \\
Shannon & 0.356 & 0.024 & 0.002 & 0.000 & 0.000 & 0.117 \\
Pielou & 0.174 & 0.156 & 0.015 & 0.000 & 0.001 & 0.931 \\
Hill_1 & 0.356 & 0.023 & 0.003 & 0.000 & 0.002 & 0.068 \\
Hill_2 & 0.121 & 0.270 & 0.194 & 0.002 & 0.004 & 0.476 \\
\hline
\end{tabular}
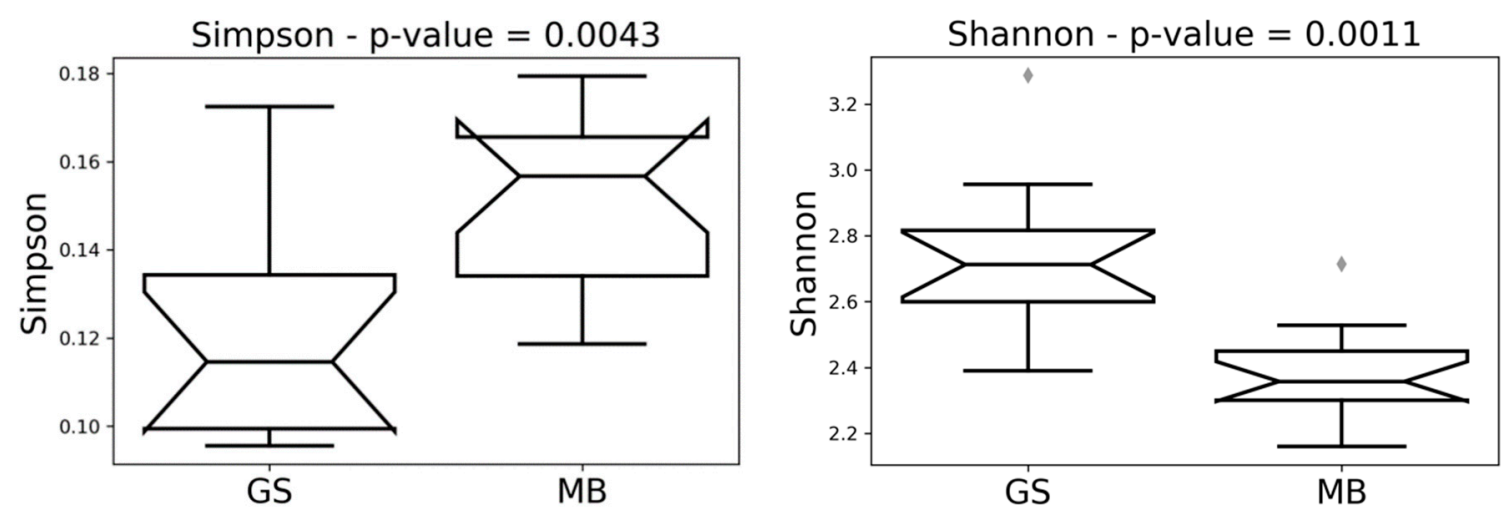

Figure 1. Cont. 

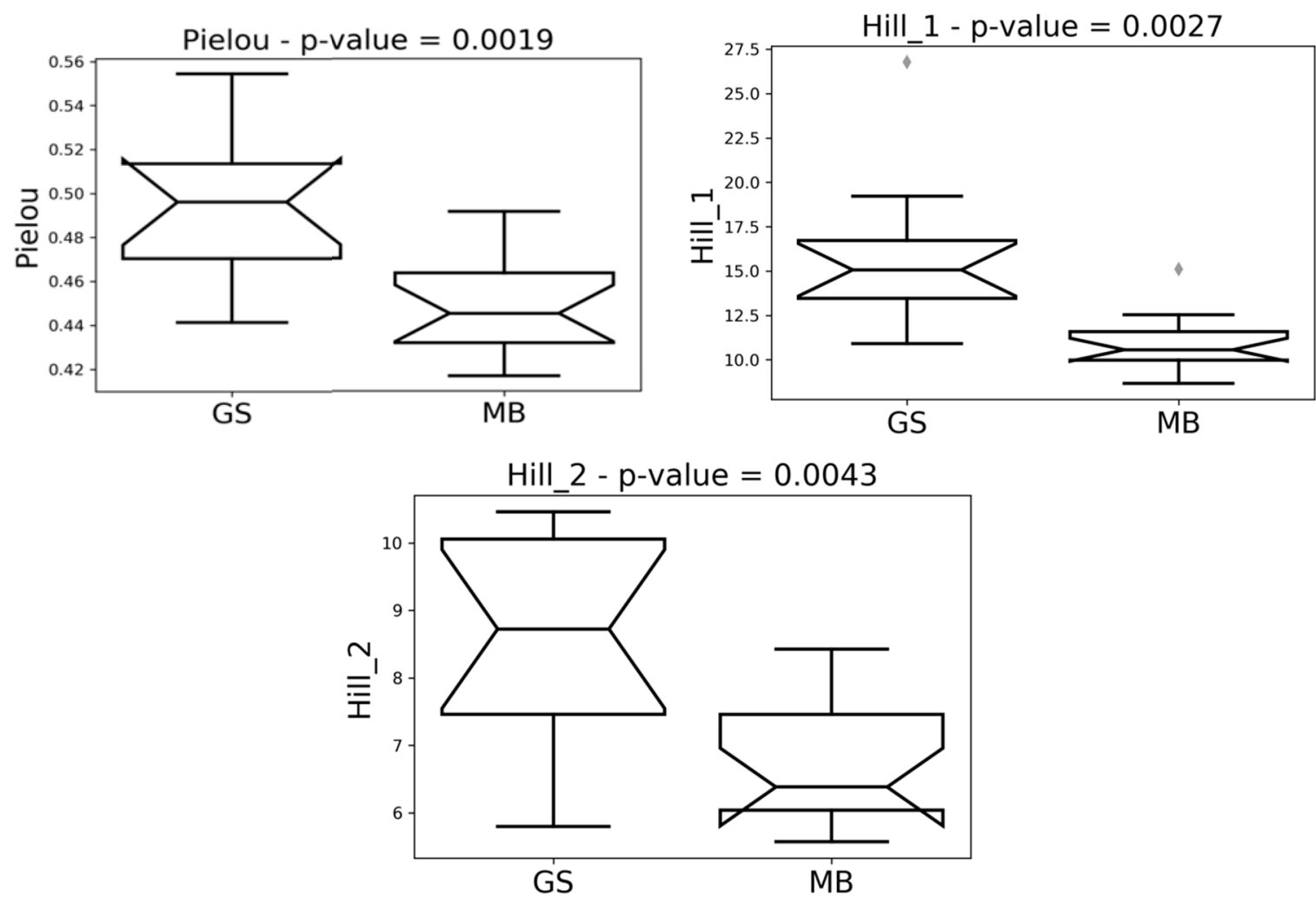

Figure 1. $p$ values alpha diversity calculated by different indexes in the tested groups. GS = German Shepherd, $\mathrm{MB}=$ mixed breeds.

Table 8. Index of median values in two groups.

\begin{tabular}{cccccc}
\hline & Simpson & Shannon & Pielou & Hill_1 & Hill_2 \\
\hline \multicolumn{5}{c}{ Phylum } \\
\hline MB & 0.446 & 1.010 & 0.420 & 2.792 & 2.382 \\
GS & 0.531 & 0.927 & 0.366 & 2.594 & 2.032 \\
\hline \multicolumn{5}{c}{ Class } \\
\hline MB & 0.296 & 1.467 & 0.486 & 4.365 & 3.454 \\
GS & 0.277 & 1.592 & 0.514 & 4.956 & 3.742 \\
\hline \multicolumn{5}{c}{ Order } \\
\hline MB & 0.296 & 1.482 & 0.388 & 4.434 & 3.456 \\
GS & 0.272 & 1.660 & 0.421 & 5.308 & 3.809 \\
\hline \multicolumn{5}{c}{ Family } \\
\hline MB & 0.171 & 2.090 & 0.462 & 8.148 & 6.021 \\
GS & 0.135 & 2.445 & 0.523 & 11.752 & 7.618 \\
\hline \multicolumn{5}{c}{ Genus } \\
\hline MB & 0.150 & 2.379 & 0.449 & 10.895 & 6.771 \\
GS & 0.122 & 2.736 & 0.496 & 15.835 & 8.511 \\
\hline \multicolumn{5}{c}{ Species } \\
\hline MB & 0.045 & 3.712 & 0.589 & 41.635 & 22.712 \\
GS & 0.065 & 3.881 & 0.590 & 50.536 & 20.544 \\
\hline
\end{tabular}




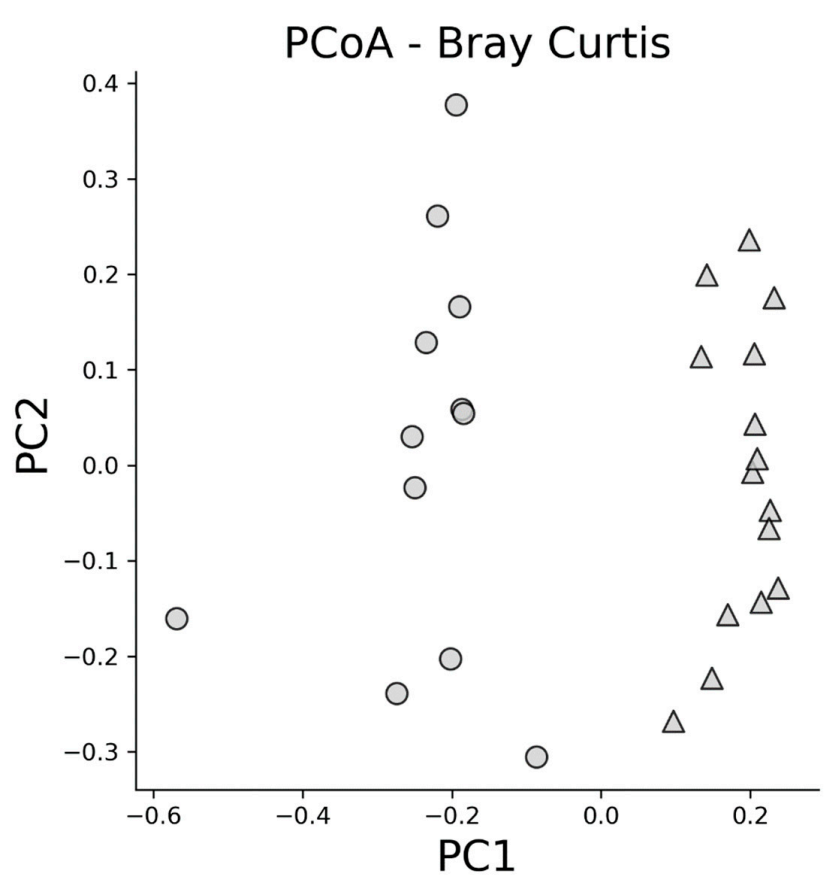

Figure 2. Beta diversity of the two study groups. Bray-Curtis beta diversity and Principal Coordinates Analysis (PCoA) the inter-sample variation highlighted a significant separation between the structural composition of the gut microbiota among study groups. ( $\mathrm{GM}=$ German Shepherd, $\mathrm{MB}=$ mixed breeds).
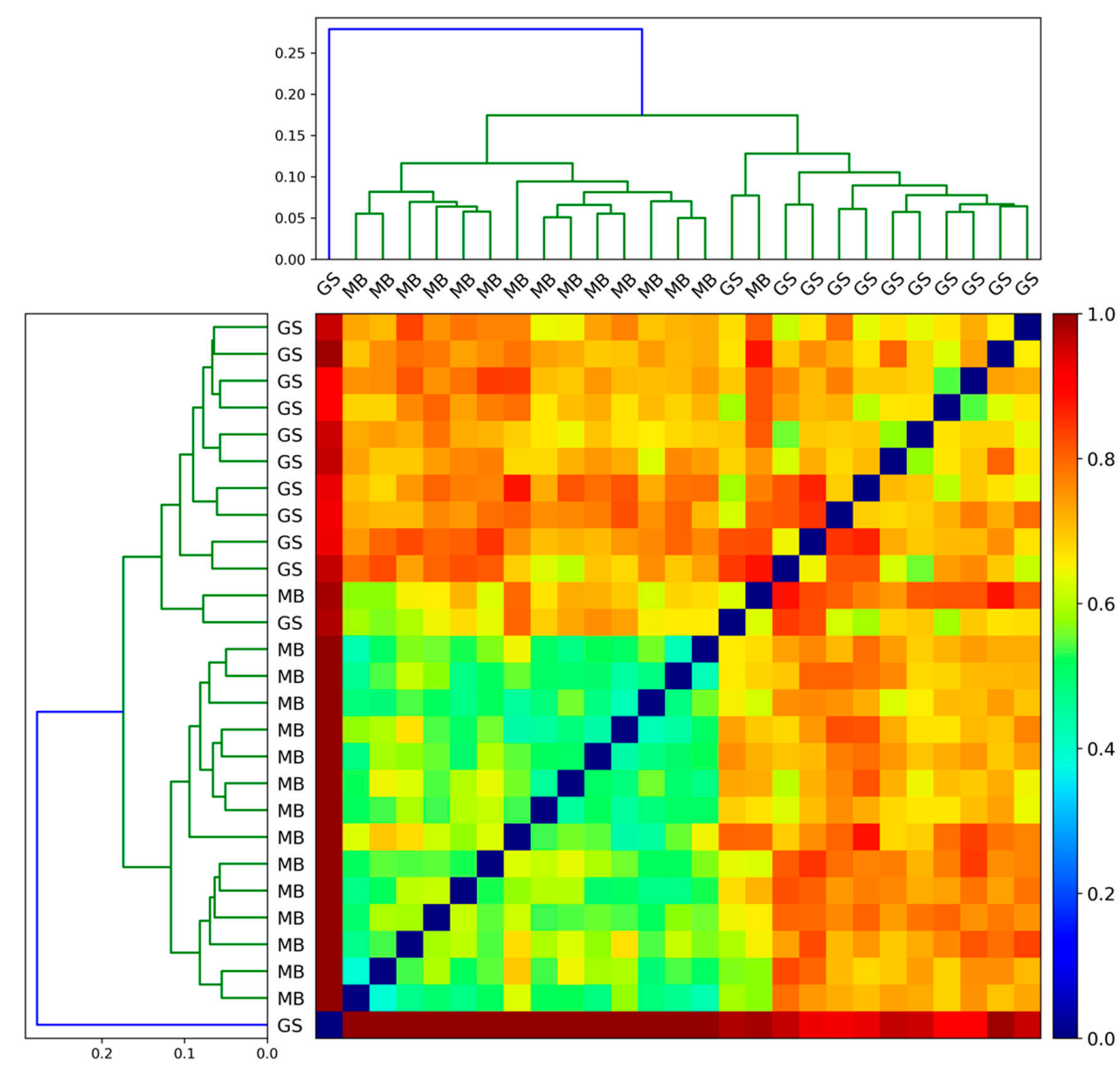

Figure 3. Heat-map diagram of the gut microbiota composition at genus level for two groups. 


\section{Discussion}

Recently, gut-microbiota plays an important role in neuroscience research as a component of the microbiota-gut-brain axis [3]. Our current knowledge about the microbiota-depression relationship is mainly based on animal model studies because very few research has been conducted on human patients. Some findings on animal models cannot fit in with the real human condition, so it can be considered as one of the most intriguing and controversial topics [16].

In this study, the relationship between the microbiota of German Shepherds and other dog breeds was investigated for the first time. In addition to that, the microbiota of the German Shepherd group was compared with that of humans suffering from depression disorder [17]. The choice of German Shepherd is due to the results derived from the quantitative molecular evaluation of fatty acids in platelets of people with mood disorders and several animals (sheep, horse, donkey, dog, cat, bovine, rat, pig and guinea pig). This investigation was conducted using a complex mathematical function, as a Self-Organizing Map (SOM) and an index (B2). This allowed classifying the different depression disorders (major depression and bipolar depression) and identifying their molecular pathway. The data obtained from animals were put into the SOM, where human disorders were classified as pathological areas, bipolar depression area and healthy human area. The results showed that sheep, cats, bovines, horses and donkeys were distributed in the opposite area on the SOM than people with mood disorders, while, guinea pigs, rats and pigs occupied the area characterized by depressive subjects. Only two different breeds of dogs (German Shepherd and Alaskan Malamute) were collocated in the part of the map typical of humans affected by Major Depression (MD). This result was not found in any other animals [13].

In the literature, the human research that associates depression and microbiota composition is controversial. In 2015, Jiang and colleagues analyzed the gut microbiota composition of humans with a diagnosis of active major depressive disorder and responded-MDD [16]. This study evidenced that both groups have an increase in genera Phascolarctobacterium, Roseburia and Parabacteroides and a decrease in genera Ruminococcus, Prevotella, Dialister and Faecalibacterium [16]. The research held by Zheng et al. (2016) [18] and Valles-Colomer et al. (2019) [19] observed an increased relative abundance of Lactobacillus in depressed patients. Furthermore, Valles-Colomer et al. (2019) [19] found a higher prevalence of Bacteroides in people suffering from depression.

In this study, the gut microbiota structure of 27 dogs (12 German Shepherd and 15 mixed breeds) was analyzed. Moreover, we have compared the gut microbiota of our German Shepherds with the gut microbiota of depressed humans, previously analyzed by Jiang et al. [16]. The phylogenetic profiles of the canine gut microbiota observed in the cohort of this research were found to be aligned with those already reported in the literature for healthy dogs $[20,21]$ but with a slightly higher abundance of Proteobacteria and a lower abundance of Bacteroidetes. According to our results, the gut microbiota of the GS group is characterized by higher fecal microbial diversity, estimated using the Shannon Index, rather than the MB group. Interestingly, gut microbiota diversity has been usually considered beneficial for human health but some studies have denied this statement [19,22]. The GS group was characterized by an enrichment of Streptococcus and Lactobacillus genera compared to the MB group. Bacteria belonging to the genus Streptococcus are known as serotonin producers, while those belonging to Lactobacillus are gamma-aminobutyric acid (GABA) producers. These molecules are recognized as neurotransmitters, able to regulate emotional behaviors [23-25]. Moreover, the GS group was characterized by a decrease in the Bacteroides genus, associated with metabolic diseases quite common in depression [26]. Furthermore, in the Firmicutes phylum, the abundance of the Ruminococcaceae family was significantly lower in the GS than in MB. Studies on mice reported a correlation between the Ruminococcaceae family and behavioral changes [27]. An overgrowth of the Gammaproteobacteria class, particularly Enterobacteriales and Enterobacteriaceae, was also detected in the GS group compared to MB. The Enterobacteriaceae includes enteric pathogens, which induce to inflammatory state and an increase of gut wall permeability, allowing bacteria to translocate 
into mesenteric lymph nodes or in the systemic circulation [28]. This leads to an increase of plasma immunoglobulin A and/or M, which is common in depressed patients [29].

The results obtained in this research represent an important step for future studies on depression, as they demonstrate that the dog can be a good animal model for mood disorders in humans [30]. The similarities between humans and dogs are due to the coexistence of these two species for over 14 thousand years [31]. The canine species might be considered as an ideal model for the study of human depression also because of the structural and functional similarities found between the canine and the human gut microbiota. Thus, in this case, the studies about dogs provide a benefit both directly for dogs and for their potential to be generalized to humans [32].

\section{Conclusions}

The results of this research demonstrate that German Shepherd's gut microbiota have a different composition compared to other dog breeds and its microbiota is like that of depressed human beings. A comparative analysis on gut-microbiota of German Shepherd and humans affected by depression showed a close resemblance in the bacterial composition that led us to talk about bacterial geography. Moreover, this study confirms the molecular affinity between German Shepherd and humans suffering from depression. We consider it appropriate to deepen these aspects in the light of the recent document about consciousness in the animal kingdom ("Cambridge Declaration on Consciousness" in 2012 (http://nonhumanrights.net/), "Document of Bologna" in 2014 (The Document of Bologna was underwritten by Bernroider, Cocchi, Gabrielli, Globus, Malik, Mender, Mullis, Pessa, Pregnolato, Pylkkanen, Rasenick, Tonello, Tuszynski, Vitiello, Werneke, Zizzi in research institute for the quantitative and quantum dynamics of living organisms on June 20,2014)). Future investigations about the interaction between gut microbiota and brain axis in animals and humans will clarify any doubts over this bidirectional communication.

Supplementary Materials: The following are available online at http://www.mdpi.com/2076-3417/10/2/573/s1, Table S1. Metagenomes tested in this study and public available in MG RAST (https://www.mg-rast.org/mgmain. html?mgpage= project\&project=mgp86824).

Author Contributions: For research articles with several authors, a short paragraph specifying their individual contributions must be provided. The following statements should be used conceptualization, E.M. and M.C.; methodology, A.D.C. and G.M. (Gerardo Manfreda); software, G.M. (Gerardo Manfreda) and A.D.C.; validation, A.D.C. and G.M. (Giovanna Marliani); formal analysis, C.S.; investigation, M.C.; data curation, A.D.C. and E.M.; writing - original draft preparation, E.M. and G.M. (Giovanna Marliani); writing-review and editing, E.M. and A.D.C.; visualization, P.A.A.; supervision, M.C.; project administration, G.C. and M.C. All authors have read and agreed to the published version of the manuscript

Funding: This research received no external funding.

Acknowledgments: We thank Christine Castellitto for her assistance in sampling.

Conflicts of Interest: Authors declare no conflict of interest.

\section{References}

1. Cocchi, M.; Tonello, L.; Gabrielli, F. The Molecular and Quantum Approach to Psychopathology and Consciousness-From Theory to Experimental Practice. In Major Depression Disorder- Cognitive and Neurobiological Mechanisms; Intechopen: London, UK, 2015; Chapter 3.

2. Accorsi, P.A.; Mondo, E.; Cocchi, M. Did you know that your animals have consciousness? J. Integr. Neurosci. 2017, 16, S3-S11. [CrossRef]

3. Cryan, J.F.; Dinan, T.G. Mind-altering microorganisms: The impact of the gut microbiota on brain and behavior. Nat. Rev. Neurosci. 2012, 13, 701-712. [CrossRef] [PubMed]

4. Sudo, N.; Chida, Y.; Aiba, Y.; Sonoda, J.; Oyama, N.; Yu, X.; Kubo, C.; Koga, Y. Postnatal microbial colonization programs the hypothalamic-pituitary-adrenal system for stress response in mice. J. Physiol. 2004, 558, 263-275. [CrossRef] [PubMed] 
5. Bravo, J.A.; Forsythe, P.; Chew, M.V.; Escaravage, E.; Savignac, H.M.; Dinan, T.G.; Bienenstock, J.; Cryan, J.F. Ingestion of Lactobacillus strain regulates emotional behavior and central GABA receptor expression in a mouse via the vagus nerve. Proc. Natl. Acad. Sci. USA 2011, 108, 16050-16055. [CrossRef] [PubMed]

6. Hsiao, E.Y.; McBride, S.W.; Hsien, S.; Sharon, G.; Hyde, E.R.; McCue, T.; Codelli, J.A.; Chow, J.; Reisman, S.E.; Petrosino, J.F.; et al. The microbiota modulates gut physiology and behavioral abnormalities associated with autism. Cell 2013, 155, 1451-1463. [CrossRef] [PubMed]

7. Carabotti, M. The Gut-Brain Axis: Interactions Between Enteric Microbiota, Central and Enteric Nervous Systems. Ann. Gastroenterol. 2014, 28, 203-209.

8. Lovelace, M.D.; Varney, B.; Sundaram, G.; Lennon, M.J.; Lim, C.K.; Jacobs, K.; Guillemin, G.J.; Brew, B.J. Recent evidence for an expanded role of the Kynurenine pathway of tryptophan metabolism in neurological diseases. Neuropharmacology 2017, 112, 373-388. [CrossRef] [PubMed]

9. Cocchi, M.; Tonello, L.; Cappello, G.; Nabacino, L.; Passi, S.; Baldini, N.; Soreca, I.; Paffetti, I.; Castrogiovanni, P.; Tarozzi, G. Biochemical Markers in Major Depression as interface between Neuronal Network and Artificial Neural Network (ANN). J. Biol. Res. 2006, 77-81. [CrossRef]

10. Benedetti, S.; Bucciarelli, S.; Canestrari, F.; Catalani, S.; Mandolini, S.; Marconi, V.; Mastrogiacomo, A.; Silvestri, R.; Tagliamonte, M.; Venanzini, R.; et al. Platelet's Fatty Acids and Differential Diagnosis of Major Depression and Bipolar Disorder through the Use of an Unsupervised Competitive-Learning Network Algorithm (SOM). OJD 2014, 3, 52-73. [CrossRef]

11. Cocchi, M.; Tonello, L.; Rasenick Mark, M. Human depression: A new approach in quantitative psychiatry. Ann. Gen. Psychiatr. 2010, 9, 25. [CrossRef]

12. Cocchi, M.; Tonello, L.; De Lucia, A.; Amato, P. Platelet and Brain Fatty Acids: A Model for the Classification of the Animals? Part 1. Int. J. Anthropol. 2009, 24, 69-76.

13. Cocchi, M.; Gabrielli, F.; Tonello, L.; Delogu, M.; Beghelli, V.; Mattioli, M.; Accorsi, P.A. Molecular Contiguity between Human and Animal Consciousness through Evolution: Some Considerations. J. Phylogen Evol. Biol. 2013, 1, 119. [CrossRef]

14. De Cesare, A.; Sirri, F.; Manfreda, G.; Moniaci, P.; Giardini, A.; Zampigna, M.; Meluzzi, A. Effect of dietary supplementation with Lactobacillus acidophilus D2/CSL (CECT 4529) on caecum microbioma and productive performance in broiler chickens. PLoS ONE 2017, 12, e0176309. [CrossRef] [PubMed]

15. De Cesare, A.; Caselli, E.; Lucchi, A.; Sala, C.; Parisi, A.; Manfreda, G.; Mazzacane, S. Impact of a probiotic-based cleaning product on the microbiological profile of broiler litters and chicken caeca microbiota. Poult 2019, 98, 3602-3610. [CrossRef]

16. Chesselet, M.F.; Carmichael, S.T. Animal Models of Neurological Disorders. Neurotherapeutics 2012, 9, 241-244. [CrossRef]

17. Jiang, H.; Ling, Z.; Mao, H.; Ma, Z.; Yin, Y.; Wang, W.; Tang, W.; Tan, Z.; Shi, J.; Li, L.; et al. Altered fecal microbiota composition in patients with major depressive disorder. Brain Behav. Immun. 2015, 48, 186-194. [CrossRef]

18. Zheng, P.; Zeng, B.; Zhou, C.; Liu, M.; Fang, Z.; Xu, X.; Zeng, L.; Chen, J.; Fan, S.; Du, X.; et al. Gut microbiome remodeling induces depressive-like behaviors through a pathway mediated by the host's metabolism. Mol. Psychiatry 2016, 21, 786-796. [CrossRef]

19. Valles-Colomer, M.; Faolony, G.; Darzi, Y.; Tigchelaar, E.F.; Wang, J.; Tito, R.L.; Schiweck, C.; Kurilshikov, A.; Joossens, M.; Vieira-Silva, S.; et al. The neuroactive potential of the human gut microbiota in quality of life and depression. Nat. Microbiol. 2019, 4, 623-632. [CrossRef]

20. Sandri, M.; Del Monego, S.; Conte, G.; Sgorlon, S.; Stefanon, B. Raw meat based diet influences faecal microbiome and end products of fermentation in healthy dogs. BMC Vet. Res. 2017, 13, 65. [CrossRef]

21. Omatsu, T.; Omura, M.; Katayama, Y.; Kimura, T.; Okumura, M.; Okumura, A.; Murata, Y.; Mizutani, T. Molecular diversity of the faecal microbiota of Toy Poodles in Japan. J. Vet. Med. Sci. 2018, 80, 749-754. [CrossRef]

22. Winter, G.; Hart, R.A.; Charlesworth, R.P.G.; Sharpley, C.F. Gut microbiome and depression: What we know and what we need to know. Rev. Neurosci. 2018, 29, 629-643. [CrossRef] [PubMed]

23. Yunes, R.A.; Poluektova, E.U.; Dyachkova, M.S.; Klimina, K.M.; Kovtun, A.S.; Averina, O.V.; Orlova, V.S.; Danilenko, V.N. GABA production and structure of gadB/gadC genes in Lactobacillus and Bifidobacterium strains from human microbiota. Anaerob 2016, 42, 197-204. [CrossRef] [PubMed] 
24. Evrensel, A.; Ceylan, M.E. The Gut-Brain Axis: The Missing Link in Depression. Clin. Psychopharmacol. Neurosci. 2015, 13, 239-244. [CrossRef]

25. Butnoriene, J.; Bunevicius, A.; Norkus, A.; Bunevicius, R. Depression but not anxiety is associated with metabolic syndrome in primary care based community sample. Psychoneuroendocrinology 2014, 40, 269-276. [CrossRef] [PubMed]

26. Bangsgaard Bendtsen, K.M.; Krych, L.; Sorensen, D.B.; Pang, W.; Nielsen, D.S.; Josefsen, K.; Hansen, L.H.; Sorensen, S.J.; Hansen, A.K. Gut microbiota composition is correlated to grid floor induced stress and behavior in the BALB/c mouse. PLoS ONE 2012, 7, e46231. [CrossRef]

27. O'Malley, D.; Julio-Pieper, M.; Gibney, S.M.; Dinan, T.G.; Cryan, J.F. Distinct alterations in colonic morphology and physiology in two rat models of enhanced stress-induced anxiety and depression-like behavior. Stress 2010, 13, 114-122. [CrossRef]

28. Maes, M.; Kubera, M.; Leunis, J.C.; Berk, M. Increased IgA and IgM responses against gut commensals in chronic depression: Further evidence for increased bacterial translocation or leaky gut. J. Affect. Disord. 2012, 141, 55-62. [CrossRef]

29. Nobis, G. Der älteste Haushund lebte vor 14,000 Jahren. Umschau 1979, 79, 610.

30. Sarviaho, R.; Hakosalo, O.; Tiira, K.; Sulkama, S.; Salmela, E.; Hytönen, M.K.; Sillanpää, M.J.; Lohi, H. Two novel genomic regions associated with fearfulness in dogs overlap human neuropsychiatric loci. Nature 2019, 9, 18. [CrossRef]

31. Udell, M.A.; Wynne, C.D. A review of domestic dogs' (Canis familiaris) human-like behaviors: Or why behavior analysts should stop worrying and love their dogs. J. Exp. Anal. Behav. 2008, 89, 247-261. [CrossRef]

32. Shafquat, A.; Joice, R.; Simmons, S.; Huttenhower, C. Functional and phylogenetic assembly of microbial communities in the human microbiome. Trends Microbiol. 2014, 22, 261-266. [CrossRef] [PubMed]

(C) 2020 by the authors. Licensee MDPI, Basel, Switzerland. This article is an open access article distributed under the terms and conditions of the Creative Commons Attribution (CC BY) license (http://creativecommons.org/licenses/by/4.0/). 